\title{
An upper bound for the waiting time for doubly nonlinear parabolic equations
}

\author{
KIANHWA C. DJIE ${ }^{\dagger}$ \\ Lehrstuhl I für Mathematik, RWTH Aachen, \\ Wüllnerstr. zw. 5 u. 7, D-52056 Aachen, Germany
}

[Received 20 January 2006 and in revised form 30 May 2006]

We obtain an upper bound for the waiting time for the doubly nonlinear parabolic equations

$$
\left\{\begin{aligned}
\left(|u|^{q-2} u\right)_{t}-\operatorname{div}\left(|\nabla u|^{p-2} \nabla u\right) & =0 & & \text { in } \mathbb{R}^{N} \times[0, \infty), \\
u(x, 0) & =u_{0}(x) & & \text { for all } x \in \mathbb{R}^{N},
\end{aligned}\right.
$$

depending on the growth of the initial value $u_{0}$ with parameters $p \geqslant 2,1<q<p$, and $\left|u_{0}\right|^{q-1} \in$ $L^{1}\left(\mathbb{R}^{N}\right)$. This upper bound coincides with the lower bound given by Giacomelli-Grün [4]. Therefore it is optimal.

Special cases are the porous medium equation (for $p=2$ ), for which we obtain the result of Chipot-Sideris [2], and the parabolic $p$-Laplace equation (for $q=2$ ).

2000 Mathematics Subject Classification: 35B05, 35B30, 35B33, 35B40, 35K55, 35K65, 35R35.

Keywords: Nonlinear degenerate parabolic equations; waiting time.

There is a wide class of equations with the property that their solutions exhibit a waiting time phenomenon, i.e. they have a strictly positive waiting time in the following sense: Let $\Omega:=$ $\operatorname{supp}\left(u_{0}\right) \subset \mathbb{R}^{N}$. Then

$$
t_{\Omega}^{\star}:=\sup \left\{t \geqslant 0 \mid u(x, \tau)=0 \text { for all } x \in \mathbb{R}^{N} \backslash \Omega \text { and } \tau \in[0, t]\right\}
$$

is called the waiting time for $u$. In particular for $x_{0} \in \partial \Omega$ we call

$t_{\Omega, x_{0}}^{\star}:=\sup \left\{t \geqslant 0 \mid\right.$ for any $\tau \in[0, t]$ there exists $\varepsilon>0$ with $u(x, \tau)=0$ for all $\left.x \in B\left(x_{0}, \varepsilon\right) \backslash \Omega\right\}$

the local waiting time for $u$ at $x_{0}$. Our aim is to achieve a quantitative upper bound for the local waiting time at $x_{0}$ depending on the growth of the initial value $u_{0}$ near $x_{0}$.

In many cases it is possible to obtain a quantitative lower bound for this waiting time as one can see in Giacomelli-Grün [4]. The recent monograph of Antontsev-Díaz-Shmarev [1] provides conditions for the appearance of waiting time by the use of energy methods, and ShishkovShchelkov [9] showed that the supports of solutions for the doubly nonlinear parabolic equation spread slowly for small initial data. But not much is known about quantitative upper bounds for the waiting time. Only for the porous medium equation (e.g. Chipot-Sideris [2]) an upper bound has been obtained, and very recently Choi-Kim [3] found conditions describing waiting time phenomena for the Hele-Shaw and Stefan problems.

${ }^{\dagger}$ E-mail: djie@math1.rwth-aachen.de 
Now, we are concerned with the class of doubly nonlinear parabolic equations

$$
\left\{\begin{aligned}
\left(|u|^{q-2} u\right)_{t}-\operatorname{div}\left(|\nabla u|^{p-2} \nabla u\right) & =0 & & \text { in } \mathbb{R}^{N} \times[0, \infty), \\
u(x, 0) & =u_{0}(x) & & \text { for all } x \in \mathbb{R}^{N},
\end{aligned}\right.
$$

with the Euclidean norm $|x|:=\left(\sum_{i=1}^{N} x_{i}^{2}\right)^{1 / 2}$ and parameters

$$
p \geqslant 2, \quad 1<q<p,
$$

and a nonnegative initial value $u_{0}: \mathbb{R}^{N} \rightarrow[0, \infty)$ which satisfies

$$
u_{0}^{q-1} \in L^{1}\left(\mathbb{R}^{N}\right)
$$

Special cases ( $p=2$ resp. $q=2$ ) are the porous medium equation and the parabolic $p$-Laplace equation.

Our strategy is to compare $u$ with a radially symmetric solution $v \leqslant u$ and to estimate the waiting time for $v$ by means of the Hardy inequality and a method introduced by Chipot-Sideris [2]. As a consequence we will obtain an upper bound for the local waiting time for $u$ which is formulated in the following Main Theorem:

THEOREM 1 (Upper bound for the waiting time) Let $u_{0} \geqslant 0, u_{0}^{q-1} \in L^{1}\left(\mathbb{R}^{N}\right)$, and let $x_{0} \in \partial \Omega$ be such that there is a ball $B=B\left(y_{0}, r_{0}\right) \subseteq \Omega$ and $A, \gamma>0$ with $x_{0} \in \partial B$ and $u_{0}(x) \geqslant A\left|x-x_{0}\right|^{\gamma}$ for all $x \in B$. Then there exists a weak solution of (1) with initial value $u_{0}$ and the following property: If $\gamma=p /(p-q)$, then $t_{\Omega, x_{0}}^{\star} \leqslant C A^{-(p-q)}$ with $C=C(p, q)>0$, independent of the spatial dimension. If $\gamma<p /(p-q)$, then $t_{\Omega, x_{0}}^{\star}=0$.

REMARK 2 The lower bound in [4] coincides with this upper bound-therefore the critical growth exponent $\gamma$ and the scaling of the waiting time with respect to $A$ turn out to be optimal. Setting $v:=|u|^{q-2} u$ one gets $v_{t}=\operatorname{div}\left(\left|\nabla v^{m}\right|^{p-2} \nabla v^{m}\right)$ with $m:=1 /(q-1)$. For $p=2$ we recover the result of Chipot-Sideris [2]. sense:

If $\left|u_{0}\right|^{q-1} \in L^{1}\left(\mathbb{R}^{N}\right)$, Ishige [6] proves the existence of a weak solution of $[1]$ in the following

Definition 3 A measurable function $u: \mathbb{R}^{N} \times[0, \infty) \rightarrow \mathbb{R}$ is called a weak solution of (1) if for all bounded open sets $\Omega \subset \mathbb{R}^{N}$ and $T>0$ we have

$$
|\nabla u|^{p-1} \in L^{1}(\Omega \times(0, T)), \quad|u|^{q-2} u \in C^{0}\left([0, \infty) ; L^{1}(\Omega)\right),
$$

and

$\int_{\Omega}|u|^{q-2} u \varphi(x, t) \mathrm{d} x-\int_{0}^{t} \int_{\Omega}\left(|u|^{q-2} u\right) \varphi_{t}+\int_{0}^{t} \int_{\Omega}|\nabla u|^{p-2} \nabla u \cdot \nabla \varphi=\int_{\Omega}\left|u_{0}\right|^{q-2} u_{0} \varphi(x, 0) \mathrm{d} x$ for all $\varphi \in W^{1, \infty}\left([0, \infty) ; L^{\infty}(\Omega)\right) \cap L^{\infty}\left([0, \infty) ; W_{0}^{1, \infty}(\Omega)\right)$ and $t>0$.

We remark immediately that there exists a radially symmetric solution for radially symmetric data. 
LEMma 4 For all $\gamma \in O(N):=\left\{A \in \mathbb{R}^{N \times N} \mid A^{T}=A^{-1}\right\}, v \geqslant 0$, and $f_{\gamma}(x):=f\left(\gamma^{-1} x\right)$ for smooth $f$ we have

$$
\operatorname{div}\left(\left(\left|\nabla u_{\gamma}\right|^{2}+v\right)^{(p-2) / 2} \nabla u_{\gamma}\right)=\left(\operatorname{div}\left(\left(|\nabla u|^{2}+v\right)^{(p-2) / 2} \nabla u\right)\right)_{\gamma} .
$$

Proof. One gets

$$
\begin{aligned}
\operatorname{div} & \left.\left(\left|\nabla u_{\gamma}\right|^{2}+v\right)^{(p-2) / 2} \nabla u_{\gamma}\right) \\
& =\nabla\left(\left|\nabla u_{\gamma}\right|^{2}+v\right)^{(p-2) / 2} \cdot \nabla u_{\gamma}+\left(\left|\nabla u_{\gamma}\right|^{2}+v\right)^{(p-2) / 2} \Delta u_{\gamma} \\
& =\nabla\left(\left|(\nabla u)_{\gamma} \gamma^{-1}\right|^{2}+v\right)^{(p-2) / 2} \cdot(\nabla u)_{\gamma} \gamma^{-1}+\left(\left|(\nabla u)_{\gamma} \gamma^{-1}\right|^{2}+v\right)^{(p-2) / 2} \sum_{j=1}^{N} \frac{\partial}{\partial x_{j}}\left(\frac{\partial}{\partial x_{j}}\left(u_{\gamma}\right)\right) \\
& =\nabla\left(\left|(\nabla u)_{\gamma}\right|^{2}+v\right)^{(p-2) / 2} \cdot(\nabla u)_{\gamma} \gamma^{-1}+\left(\left|(\nabla u)_{\gamma}\right|^{2}+v\right)^{(p-2) / 2} \sum_{j, l=1}^{N} \frac{\partial}{\partial x_{j}}\left(\left(\frac{\partial u}{\partial x_{l}}\right)_{\gamma}\right) \cdot \gamma_{j l} \\
& =\nabla\left(\left(|\nabla u|^{2}+v\right)_{\gamma}^{(p-2) / 2}\right) \cdot(\nabla u)_{\gamma} \gamma^{-1}+\left(|\nabla u|^{2}+v\right)_{\gamma}^{(p-2) / 2} \sum_{j, l, m=1}^{N}\left(\frac{\partial^{2} u}{\partial x_{l} \partial x_{m}}\right)_{\gamma} \cdot\left(\gamma_{j m} \gamma_{j l}\right) \\
& =\left(\nabla\left(|\nabla u|^{2}+v\right)^{(p-2) / 2}\right)_{\gamma} \gamma^{-1} \cdot(\nabla u)_{\gamma} \gamma^{-1}+\left(|\nabla u|^{2}+v\right)_{\gamma}^{(p-2) / 2} \sum_{l, m=1}^{N}\left(\frac{\partial^{2} u}{\partial x_{l} \partial x_{m}}\right)_{\gamma} \cdot \delta_{m l} \\
& =\left(\nabla\left(|\nabla u|^{2}+v\right)^{(p-2) / 2}\right)_{\gamma} \cdot(\nabla u)_{\gamma}+\left(|\nabla u|^{2}+v\right)_{\gamma}^{(p-2) / 2}(\Delta u)_{\gamma} \\
& =\left(\nabla\left(|\nabla u|^{2}+v\right)^{(p-2) / 2} \cdot \nabla u+\left(|\nabla u|^{2}+v\right)^{(p-2) / 2}(\Delta u)\right)_{\gamma} \\
& =\left(\operatorname{div}\left(\left(|\nabla u|^{2}+v\right)^{(p-2) / 2} \nabla u\right)\right)_{\gamma},
\end{aligned}
$$

since the orthogonal matrix $\gamma^{-1}$ induces an isometry as a linear map.

THEOREM 5 (Existence of a radially symmetric weak solution) Let $u_{0} \geqslant 0$ be radially symmetric with nonpositive radial derivative $\left(u_{0}\right)_{r} \leqslant 0$. Then there exists a radially symmetric weak solution $u \geqslant 0$ of (1) with initial value $u_{0}$ and $u_{r} \leqslant 0$.

Proof. This is a consequence of the existence theorem in [6, 11]. We will sketch the additional arguments.

Let $u_{0} \in C_{0}^{\infty}(\Omega)$ for $\Omega:=B(0, n)$ and $M:=\max \left\{u_{0}(x) \mid x \in \Omega\right\}$ in a first step (which will approximate our general $u_{0}$ ). Consider the initial-boundary value problem

$$
\left\{\begin{aligned}
\left(|u|^{q-2} u\right)_{t}-\operatorname{div}\left(\left(|\nabla u|^{2}+v\right)^{(p-2) / 2} \nabla u\right) & =0 & & \text { for }(x, t) \in \Omega \times(0, \infty), \\
u(x, t) & =\varepsilon & & \text { for }(x, t) \in \partial \Omega \times[0, \infty), \\
u(x, 0) & =u_{0}(x)+\varepsilon & & \text { for } x \in \Omega,
\end{aligned}\right.
$$

for any $\varepsilon, v \in(0,1]$, and choose a smooth monotone function $\varphi_{\varepsilon} \in C^{\infty}(\mathbb{R})$ with

$$
\varphi_{\varepsilon}(z)= \begin{cases}(\varepsilon / 2)^{2-q} & \text { for } z \leqslant \varepsilon / 2 \\ z^{2-q} & \text { for } z \in[\varepsilon, M+\varepsilon] \\ (M+2 \varepsilon)^{2-q} & \text { for } z \geqslant M+2 \varepsilon\end{cases}
$$


Then we first examine the regularized problem

$$
\left\{\begin{aligned}
u_{t}-\frac{1}{q-1} \varphi_{\varepsilon}(u) \operatorname{div}\left(\left(|\nabla u|^{2}+v\right)^{(p-2) / 2} \nabla u\right) & =0 & & \text { for }(x, t) \in \Omega \times(0, \infty), \\
u(x, t) & =\varepsilon & & \text { for }(x, t) \in \partial \Omega \times[0, \infty), \\
u(x, 0) & =u_{0}(x)+\varepsilon & & \text { for } x \in \Omega .
\end{aligned}\right.
$$

The differential equation (3) has the form

$$
u_{t}-\sum_{i, j=1}^{N} A_{i j}(x, t, u, \nabla u) u_{x_{i} x_{j}}=0
$$

with $A=\left(A_{i j}\right)_{i, j=1, \ldots, n}:=B+C$ and

$$
\begin{aligned}
B(x, t, z, \eta) & :=\frac{p-2}{q-1} \varphi_{\varepsilon}(z)\left(|\eta|^{2}+v\right)^{(p-2) / 2-1} \cdot\left(\eta \cdot \eta^{T}\right), \\
C(x, t, z, \eta) & :=\frac{1}{q-1} \varphi_{\varepsilon}(z)\left(|\eta|^{2}+v\right)^{(p-2) / 2} I_{N}
\end{aligned}
$$

for $x \in \Omega, t \in[0, \infty), z \in \mathbb{R}$, and $\eta \in \mathbb{R}^{N}$. This equation has a unique classical solution $u_{\varepsilon, \nu} \in \bigcap_{T>0} \bigcap_{\beta>0} H^{2+\beta, 1+\beta / 2}(\overline{\Omega \times[0, T)})$ due to Theorem VI.4.1 of [7]-with notation $H^{2+\beta, 1+\beta / 2}(\overline{\Omega \times[0, T)})$ from page 7 of [7]. Then $u_{\varepsilon, v}$ is a classical solution of 2 , because $\varepsilon \leqslant u_{\varepsilon, v} \leqslant M+\varepsilon$ by comparison; see e.g. Theorem 3.12 of [8].

Due to the radially symmetric initial value and Lemma $4 .\left(u_{\varepsilon, v}\right)_{\gamma}$ is also a solution of $\sqrt{3}$ for all $\gamma \in O(N)$. Therefore we obtain $u_{\varepsilon, v}=\left(u_{\varepsilon, v}\right)_{\gamma}$ from the uniqueness. Hence $u_{\varepsilon, v}$ is radially symmetric, that is, $u_{\varepsilon, v}(x, t)=\tilde{u}(|x|, t)$ for $\tilde{u}=\tilde{u}(r, t):[0, n] \times[0, \infty) \rightarrow[\varepsilon, M+\varepsilon]$. As $u$ is smooth (in particular at the origin), it follows that $\frac{\partial^{k} \widetilde{u}}{\partial r^{k}}(0, t)=0$ for all $k \in \mathbb{N}$ and $t \in[0, \infty)$.

Now $\tilde{u}$ is a classical solution for every $T>0$ of the following differential equation:

$$
\left\{\begin{aligned}
\left(\tilde{u}^{q-1}\right)_{t} r^{N-1}-\left[\left(\left(\tilde{u}_{r}\right)^{2}+v\right)^{(p-2) / 2} r^{N-1} \tilde{u}_{r}\right]_{r} & =0, & & (r, t) \in(0, n) \times(0, T], \\
\tilde{u}(r, t) & =u_{\varepsilon, v}(r, t), & & r \in\{0, n\}, t \in[0, T], \\
\tilde{u}(r, 0) & =\tilde{u}_{0}(r)+\varepsilon, & & r \in(0, n),
\end{aligned}\right.
$$

which has the form

$$
\tilde{u}_{t}-a_{11}\left(r, t, \widetilde{u}_{r}\right) \tilde{u}_{r r}+a\left(r, t, \widetilde{u}_{r}\right)=0
$$

with

$$
\begin{aligned}
a_{11}(r, t, \eta) & :=\frac{1}{q-1} \varphi_{\varepsilon}(\widetilde{u}(r, t))\left(\eta^{2}+v\right)^{(p-2) / 2-1}\left[(p-1) \eta^{2}+v\right]>0, \\
a(r, t, \eta) & :=-\frac{N-1}{q-1} \cdot \frac{\tilde{u}_{r}(r, t)}{r} \cdot \varphi_{\varepsilon}(\widetilde{u}(r, t))\left(\eta^{2}+v\right)^{(p-2) / 2}
\end{aligned}
$$

for $r \in(0, n), t \in(0, T]$ and $z, \eta \in \mathbb{R}$. Now, $\tilde{u}_{r}(n, t) \leqslant 0$, because $\widetilde{u}(n, t)=\varepsilon \leqslant \widetilde{u}$. Differentiating (4) with respect to $r$ leads to

$$
\left\{\begin{array}{rlrl}
\left(\tilde{u}_{r}\right)_{t}-a_{11}\left(r, t, \tilde{u}_{r}\right)\left(\tilde{u}_{r}\right)_{r r}+b\left(r, t, \tilde{u}_{r},\left(\tilde{u}_{r}\right)_{r}\right) & =0, & & (r, t) \in(0, n) \times(0, T], \\
\tilde{u}_{r}(r, t) \leqslant 0, & & r \in\{0, n\}, t \in[0, T], \\
\tilde{u}_{r}(r, 0)=\left(\widetilde{u}_{0}\right)_{r}(r) \leqslant 0, & r \in(0, n),
\end{array}\right.
$$


with

$$
b\left(r, t, \tilde{u}_{r},\left(\tilde{u}_{r}\right)_{r}\right)=\left(a\left(r, t, \tilde{u}_{r}\right)\right)_{r}-\left(a_{11}\left(r, t, \tilde{u}_{r}\right)\right)_{r}\left(\tilde{u}_{r}\right)_{r}
$$

hence

$$
\begin{aligned}
b(r, t, z, \eta):= & -\frac{N-1}{q-1}\left(z^{2}+v\right)^{(p-2) / 2-1}\left[\left(\frac{\eta}{r}-\frac{z}{r^{2}}\right) \varphi_{\varepsilon}(\widetilde{u}(r, t))\left(z^{2}+v\right)\right. \\
& \left.+\frac{\widetilde{u}_{r}(r, t)}{r}\left(z^{2}+v\right) \varphi_{\varepsilon}^{\prime}(\widetilde{u}(r, t)) z+\frac{\widetilde{u}_{r}(r, t)}{r} \varphi_{\varepsilon}(\widetilde{u}(r, t))(p-2) z \eta\right] \\
& -\frac{1}{q-1}\left(z^{2}+v\right)^{(p-2) / 2-2} \eta\left[\varphi_{\varepsilon}^{\prime}(\widetilde{u}(r, t)) z\left(z^{2}+v\right)\left((p-1) z^{2}+v\right)\right. \\
& \left.+\varphi_{\varepsilon}(\widetilde{u}(r, t)) z \eta\left((p-1)(p-2) z^{2}+3(p-2) v\right)\right] .
\end{aligned}
$$

It follows that

$$
\tilde{u}_{r}(r, t) \leqslant \max \left\{\max \left\{0, \tilde{u}_{r}(\gamma, \tau)\right\} \mid \tau \in[0, T]\right\} \quad \text { for all }(r, t) \in[\gamma, n] \times[0, T]
$$

for any $\gamma \in(0, n)$ by comparison, e.g. Theorem 3.12 of [8]. Hence $\tilde{u}_{r} \leqslant 0$ in $[0, n] \times[0, T]$ by letting $\gamma \searrow 0$ and by the uniform continuity of $\tilde{u}_{r}$.

From the arguments on page 202 of [11], one can pass to the limit as $\varepsilon, v \searrow 0$ (for a suitable subsequence) to obtain a radially symmetric nonnegative weak solution $u$ of (2) (with $\varepsilon=v=0$ ) in $\Omega$ with $\widetilde{u}_{r} \leqslant 0$.

Approximation of the general $u_{0}$ by $u_{0, n} \in C_{0}^{\infty}(B(0, n))$ as in [6] leads to a radially symmetric nonnegative weak solution $u$ of $[1]$ with $\tilde{u}_{r} \leqslant 0$.

THEOREM 6 Let $v_{0} \geqslant u_{0} \geqslant 0$. Then there exist weak solutions $v, u$ of 11 with initial values $v_{0}$ resp. $u_{0}$ such that $v \geqslant u \geqslant 0$. For $x_{0} \in \partial \operatorname{supp}\left(u_{0}\right) \cap \partial \operatorname{supp}\left(v_{0}\right)$ the local waiting times satisfy

$$
t_{\operatorname{supp}\left(v_{0}\right), x_{0}}^{\star}(v) \leqslant t_{\operatorname{supp}\left(u_{0}\right), x_{0}}^{\star}(u) .
$$

If $u_{0}$ is radially symmetric with nonpositive radial derivative, then we may assume that $u$ has the same property.

Proof. The comparison principle (e.g. Theorem 3.12 of [8]) applies to the regularized solutions of (3). Now, pass to the limit by [11, 6].

The following lemma generalizes Lemma 2 of [2].

Lemma 7 Let $A, B>0, C \geqslant 0, t^{\star} \in(0, \infty], \gamma \in \mathbb{N}_{0}, \varepsilon>1, \delta<1+(\varepsilon-1)(\gamma+1)$, and $F \in C^{0}\left(\left[0, t^{\star}\right)\right) \cap C^{1}\left(\left(0, t^{\star}\right)\right)$ with $F(0)=0, F(\tau) \geqslant 0$ for all $\tau \in\left(0, t^{\star}\right)$ and

$$
F^{\prime}(\tau) \geqslant A \tau^{\gamma}+B \tau^{-\delta} F(\tau)^{\varepsilon}+C F(\tau)
$$

Then

$$
t^{\star} \leqslant \frac{K}{C} \ln \left(1+K C\left(A^{\varepsilon-1} B\right)^{-\frac{1}{1-\delta+(\gamma+1)(\varepsilon-1)}}\right) \quad \text { with } K=K(\gamma, \delta, \varepsilon)>0
$$

For $C=0$ this should be read as $t^{\star} \leqslant K\left(A^{\varepsilon-1} B\right)^{-\frac{1}{1-\delta+(\gamma+1)(\varepsilon-1)}}$. 
Proof. Without loss of generality we may assume $t^{\star}<\infty$ by an indirect argument. By induction we prove $F(\tau) \geqslant A \tau^{\gamma+1} \sum_{k=1}^{n} \frac{1}{\prod_{i=1}^{k}(\gamma+i)}(C \tau)^{k-1}$. This is trivial for $n=0$. If it is true for $n$, then

$$
F^{\prime}(\tau) \geqslant A \tau^{\gamma}+C F(\tau) \geqslant A \tau^{\gamma}+A C \tau^{\gamma+1} \sum_{k=1}^{n} \frac{1}{\prod_{i=1}^{k}(\gamma+i)}(C \tau)^{k-1} .
$$

The induction step follows by integration.

Hence $F(\tau) \geqslant \frac{A}{\gamma+1} \tau^{\gamma+1}$ and therefore $F(\tau)>0$ for $\tau \in\left(0, t^{\star}\right)$. Simplifying $F$ leads to

$$
\begin{aligned}
F(\tau) & \geqslant A \tau^{\gamma+1} \sum_{k=0}^{\infty} \frac{1}{\prod_{i=1}^{k+1}(\gamma+i)}(C \tau)^{k}=\gamma ! A \tau^{\gamma+1} \frac{e^{C \tau}-\sum_{k=0}^{\gamma}(C \tau)^{k} / k !}{(C \tau)^{\gamma+1}} \\
& \geqslant K A \tau^{\gamma+1} \frac{e^{C \tau}}{(C \tau)^{\gamma+1}+1} \geqslant K A \tau^{\gamma+1} e^{C \tau / 2} .
\end{aligned}
$$

Choose an arbitrary $v=v(\gamma, \delta, \varepsilon) \in\left(0, \min \left\{\varepsilon-1, \varepsilon-1-\frac{\delta-1}{\gamma+1}\right\}\right)$. Then it follows that

$$
F^{\prime}(\tau) \geqslant F(\tau)^{1+\nu}\left[B \tau^{-\delta} F(\tau)^{\varepsilon-1-\nu}\right] \geqslant K F(\tau)^{1+\nu}\left[A^{\varepsilon-1-\nu} B \tau^{-\delta+(\gamma+1)(\varepsilon-1-\nu)}\right] .
$$

Since $F(\tau)>0$, we have $-\frac{1}{v}\left(F^{-v}\right)^{\prime}(\tau)=F(\tau)^{-1-v} F^{\prime}(\tau) \geqslant K A^{\varepsilon-1-v} B \tau^{-\delta+(\gamma+1)(\varepsilon-1-v)}$ for all $\tau \in\left(0, t^{\star}\right)$. Now, choose $\alpha \in(0,1), \beta \in(\alpha, 1)$, and integrate over $\left[\alpha t^{\star}, \beta t^{\star}\right]$ to obtain

$$
\begin{aligned}
\frac{1}{v} F\left(\alpha t^{\star}\right)^{-v} & \geqslant \frac{1}{v}\left[F\left(\alpha t^{\star}\right)^{-v}-F\left(\beta t^{\star}\right)^{-v}\right]=\int_{\alpha t^{\star}}^{\beta t^{\star}}-\frac{1}{v}\left(F^{-v}\right)^{\prime}(\tau) \mathrm{d} \tau \\
& \geqslant K A^{\varepsilon-1-v} B \int_{\alpha t^{\star}}^{\beta t^{\star}} \tau^{-\delta+(\gamma+1)(\varepsilon-1-v)} \mathrm{d} \tau,
\end{aligned}
$$

hence

$$
\begin{aligned}
\frac{1}{v} F\left(\alpha t^{\star}\right)^{-v} & \geqslant K A^{\varepsilon-1-v} B \int_{\alpha t^{\star}}^{t^{\star}} \tau^{-\delta+(\gamma+1)(\varepsilon-1-v)} \mathrm{d} \tau \\
& =K A^{\varepsilon-1-v} B\left(t^{\star}\right)^{1-\delta+(\gamma+1)(\varepsilon-1-v)}\left(1-\alpha^{1-\delta+(\gamma+1)(\varepsilon-1-v)}\right),
\end{aligned}
$$

since $\beta \in(\alpha, 1)$ was arbitrary. From $F\left(\alpha t^{\star}\right) \geqslant K A\left(\alpha t^{\star}\right)^{\gamma+1} e^{C \alpha t^{\star} / 2}$ it follows that

$$
\frac{1}{v}\left[K A\left(\alpha t^{\star}\right)^{\gamma+1} e^{C \alpha t^{\star} / 2}\right]^{-v} \geqslant K A^{\varepsilon-1-v} B\left(t^{\star}\right)^{1-\delta+(\gamma+1)(\varepsilon-1-\nu)}\left(1-\alpha^{1-\delta+(\gamma+1)(\varepsilon-1-v)}\right),
$$

hence

$$
\left(t^{\star}\right)^{1-\delta+(\gamma+1)(\varepsilon-1)} e^{C \nu \alpha t^{\star} / 2} \leqslant \frac{K}{A^{\varepsilon-1} B},
$$

which completes the proof for the case $C=0$. We may therefore assume $C>0$. Let $W$ denote the Lambert $W$ function, i.e. the inverse function for $x \mapsto x e^{x}$. Then we may rewrite the last inequality as

$$
t^{\star} \leqslant \frac{2(1-\delta+(\gamma+1)(\varepsilon-1))}{C \nu \alpha} W\left(\frac{C \nu \alpha}{2(1-\delta+(\gamma+1)(\varepsilon-1))}\left(\frac{K}{A^{\varepsilon-1} B}\right)^{\frac{1}{1-\delta+(\gamma+1)(\varepsilon-1)}}\right) .
$$

We have $W(x) \leqslant \ln (1+x)$, because $\ln (1+x) \geqslant \frac{x}{1+x}$ for all $x \geqslant 0$. 
We recall the well-known (for $s>1$ ) Hardy inequality.

THEOREM 8 (Hardy inequality in the cases $0<r<s, s \geqslant 1,[5,10]$ ) Let $f \in A C([a, b])$ with $f(a)=0$ and let $u, v:(a, b) \rightarrow \mathbb{R}^{>0}$ be measurable. Let $s \geqslant 1$ and $0<r<s$ arbitrary. If either $s=1$ and the integral $\int_{x}^{b} u(t) \mathrm{d} t<\infty$ is finite for all $x \in(a, b)$ and

$$
A:=\left(\int_{a}^{b}[\underset{a<t<x}{\operatorname{essinf}} v(t)]^{-\frac{r}{1-r}}\left(\int_{x}^{b} u(t) \mathrm{d} t\right)^{\frac{r}{1-r}} u(x) \mathrm{d} x\right)^{\frac{1-r}{r}}<\infty
$$

or $s>1$ and

$$
A:=\left(\int_{a}^{b}\left(\int_{x}^{b} u(t) \mathrm{d} t\right)^{\frac{s}{s-r}}\left(\int_{a}^{x} v(t)^{-\frac{1}{s-1}} \mathrm{~d} t\right)^{\frac{s(r-1)}{s-r}} v(x)^{-\frac{1}{s-1}} \mathrm{~d} x\right)^{\frac{s-r}{r s}}<\infty,
$$

then the Hardy inequality

$$
\left(\int_{a}^{b}|f(x)|^{r} u(x) \mathrm{d} x\right)^{1 / r} \leqslant C\left(\int_{a}^{b}\left|f^{\prime}(x)\right|^{s} v(x) \mathrm{d} x\right)^{1 / s}
$$

is valid with $C=C(r, s) \cdot A<\infty$.

Now, we are able to estimate the waiting time for a nonnegative radially symmetric weak solution with nonpositive radial derivative.

THEOREM 9 (Radially symmetric version) Let $R>0$ and $u: \mathbb{R}^{N} \times[0, \infty) \rightarrow[0, \infty)$ be a nonnegative radially symmetric weak solution for (1) with $\Omega=B(0, R), u(x, t)=: \widetilde{u}(|x|, t)$, and $\widetilde{u}_{r} \leqslant 0$. Let $\delta \in(1, p-1)$ for $p>2$, and $\delta:=1$ for $p=2$. Then there is a constant $C=C(p, q, \delta)>0$, independent of the spatial dimension, such that for any $\varepsilon \in(0, R)$ the estimate

$$
t_{\Omega}^{\star} \leqslant C(p, q, \delta) C\left(u_{0} ; \delta, \varepsilon\right)^{-\frac{p-q}{q-1}}\left(\frac{R}{R-\varepsilon}\right)^{\frac{(N-1)(p-1)}{q-1}}(R-\varepsilon)^{\frac{(N-1)(p-q)}{q-1}} \varepsilon^{p-1-\delta+\frac{(p-1)(\delta+1)}{q-1}}
$$

is valid with

$$
C\left(u_{0} ; \delta, \varepsilon\right):=\int_{R-\varepsilon}^{R} \widetilde{u}_{0}(r)^{q-1}\left(\varepsilon^{\delta}-(R-r)^{\delta}\right) r^{N-1} \mathrm{~d} r .
$$

Proof. Choose an arbitrary $\tau \in\left(0, t_{\Omega}^{\star}\right)$. Then we test the differential equation with the function $\varphi(x, t):=g(t) h(x)$ with $g(t):=(\tau-t)_{+}^{\gamma}$ (the value of $\gamma$ is irrelevant) and $h(x):=\widetilde{h}(|x|)$ with $\widetilde{h}(r):=\left(\varepsilon^{\delta}-|R-r|^{\delta}\right)_{+}$. Let $\Omega_{\varepsilon}:=B(0, R) \backslash B(0, R-\varepsilon)$. Then we have

$$
\begin{aligned}
\int_{\Omega_{\varepsilon}} u_{0}^{q-1} \varphi(x, 0) \mathrm{d} x+\int_{\Omega_{\varepsilon}} \int_{0}^{\tau} u^{q-1} \varphi_{t} \mathrm{~d} t \mathrm{~d} x= & {\left[\int_{\Omega_{\varepsilon}} h u_{0}^{q-1} \mathrm{~d} x\right] \tau^{\gamma}+\int_{0}^{\tau} g_{t} \int_{\Omega_{\varepsilon}} u^{q-1} h \mathrm{~d} x \mathrm{~d} t } \\
= & N \omega_{N}\left[\int_{R-\varepsilon}^{R} \widetilde{h} \widetilde{u}_{0}^{q-1} r^{N-1} \mathrm{~d} r\right] \tau^{\gamma} \\
& +N \omega_{N} \int_{0}^{\tau} g_{t} \int_{R-\varepsilon}^{R} \widetilde{u}^{q-1} \widetilde{h} r^{N-1} \mathrm{~d} r \mathrm{~d} t
\end{aligned}
$$


where $\omega_{N}$ denotes the volume of the $N$-dimensional unit ball. Moreover

$$
\frac{\partial}{\partial x_{j}} u(x, t)=\frac{\partial}{\partial x_{j}} \widetilde{u}(|x|, t)=\tilde{u}_{r}(|x|, t) \frac{x_{j}}{|x|}, \quad \text { hence } \quad \nabla u=\tilde{u}_{r}(|x|, t) \frac{x}{|x|} .
$$

Since $\nabla h(x)=\tilde{h}^{\prime}(|x|) \frac{x}{|x|}$, we obtain

$$
\begin{aligned}
-\int_{0}^{\tau} \int_{\Omega_{\varepsilon}}|\nabla u|^{p-2} \nabla u \cdot \nabla \varphi \mathrm{d} x \mathrm{~d} t & =-\int_{0}^{\tau} g \int_{\Omega_{\varepsilon}}|\nabla u|^{p-2} \nabla u \cdot \nabla h \mathrm{~d} x \mathrm{~d} t \\
& =-\int_{0}^{\tau} g \int_{\Omega_{\varepsilon}}\left|\widetilde{u}_{r}\right|^{p-2} \widetilde{u}_{r} \widetilde{h}^{\prime}(|x|) \mathrm{d} x \mathrm{~d} t \\
& =\int_{0}^{\tau} g \int_{\Omega_{\varepsilon}}\left|\tilde{u}_{r}\right|^{p-1}\left|\tilde{h}^{\prime}(|x|)\right| \mathrm{d} x \mathrm{~d} t \\
& =N \omega_{N} \int_{0}^{\tau} g \int_{R-\varepsilon}^{R}\left|\tilde{u}_{r}\right|^{p-1}\left|\tilde{h}^{\prime}\right| r^{N-1} \mathrm{~d} r \mathrm{~d} t .
\end{aligned}
$$

Therefore we have the following equality:

$$
\int_{0}^{\tau} g_{t} \int_{R-\varepsilon}^{R} \tilde{u}^{q-1} \tilde{h} r^{N-1} \mathrm{~d} r \mathrm{~d} t+\int_{0}^{\tau} g \int_{R-\varepsilon}^{R}\left|\tilde{u}_{r}\right|^{p-1}\left|\widetilde{h}^{\prime}\right| r^{N-1} \mathrm{~d} r \mathrm{~d} t+C\left(u_{0} ; \delta, \varepsilon\right) \tau^{\gamma}=0 .
$$

Now, apply the Hardy inequality with exponents $s:=p-1, r:=q-1$, and $u(t):=\tilde{h}(R-t)$. $(R-t)^{N-1}, v(t):=\left|\widetilde{h}^{\prime}(R-t)\right| \cdot(R-t)^{N-1}, t \in(0, \varepsilon)$, to the inner integral in the second summand.

In the case $p=2$ we have (with $\delta=1$ ) the estimate

$$
\begin{aligned}
\left(\int_{0}^{\varepsilon}\left[\underset{0<t<x}{\operatorname{essinf}}(R-t)^{N-1}\right]^{-\frac{q-1}{2-q}}\right. & {\left.\left[\int_{x}^{\varepsilon}(\varepsilon-t)(R-t)^{N-1} \mathrm{~d} t\right]^{\frac{q-1}{2-q}}(\varepsilon-x)(R-x)^{N-1} \mathrm{~d} x\right)^{\frac{2-q}{q-1}} } \\
& \leqslant\left(\int_{0}^{\varepsilon}(R-x)^{-\frac{(N-1)(q-1)}{2-q}}\left[\int_{x}^{\varepsilon} \varepsilon R^{N-1} \mathrm{~d} t\right]^{\frac{q-1}{2-q}} \varepsilon R^{N-1} \mathrm{~d} x\right)^{\frac{2-q}{q-1}} \\
& \leqslant(R-\varepsilon)^{-(N-1)} R^{N-1+\frac{(N-1)(2-q)}{q-1}} \varepsilon^{\left(1+\frac{2(q-1)}{2-q}+1\right) \frac{2-q}{q-1}} \\
& =\left(\frac{R}{R-\varepsilon}\right)^{\frac{N-1}{q-1}}(R-\varepsilon)^{\frac{(N-1)(p-q)}{(q-1)(p-1)}} \varepsilon^{\frac{\delta+1}{q-1}+\frac{p-1-\delta}{p-1}} .
\end{aligned}
$$

In the case $p>2$ we estimate the value $A$ (see Theorem 8 ) by

$$
\begin{gathered}
C\left(\int_{0}^{\varepsilon}\left(\int_{x}^{\varepsilon} \varepsilon^{\delta} R^{N-1} \mathrm{~d} t\right)^{\frac{p-1}{p-q}}\left(\int_{0}^{x} t^{-\frac{\delta-1}{p-2}}(R-t)^{-\frac{N-1}{p-2}} \mathrm{~d} t\right)^{\frac{(p-1)(q-2)}{p-q}} x^{-\frac{\delta-1}{p-2}}(R-x)^{-\frac{N-1}{p-2}} \mathrm{~d} x\right)^{\frac{p-q}{(p-1)(q-1)}} \\
\leqslant C R^{\frac{N-1}{q-1}}(R-\varepsilon)^{\frac{(N-1)(p-q)}{(q-1)(p-1)}\left[-\frac{(p-1)(q-2)}{(p-2)(p-q)}-\frac{1}{p-2}\right]} \varepsilon^{\left[(\delta+1) \frac{p-1}{p-q}+\left(1-\frac{\delta-1}{p-2}\right) \frac{(p-1)(q-2)}{p-q}-\frac{\delta-1}{p-2}+1\right] \frac{p-q}{(p-1)(q-1)}} \\
\quad=C\left(\frac{R}{R-\varepsilon}\right)^{\frac{N-1}{q-1}}(R-\varepsilon)^{\frac{(N-1)(p-q)}{(q-1)(p-1)}} \varepsilon^{\frac{\delta+1}{q-1}+\frac{p-1-\delta}{p-1}}
\end{gathered}
$$

where $C=C(p, q, \delta)$ denotes a generic constant depending only on $p, q, \delta$. 
Therefore in both cases we get from the Hardy inequality

$$
\begin{aligned}
& \left(\int_{R-\varepsilon}^{R}|\widetilde{u}|^{q-1} \tilde{h} r^{N-1}\right)^{\frac{1}{q-1}} \\
& \quad \leqslant C\left(\frac{R}{R-\varepsilon}\right)^{\frac{N-1}{q-1}}(R-\varepsilon)^{\frac{(N-1)(p-q)}{(q-1)(p-1)}} \varepsilon^{\frac{\delta+1}{q-1}+\frac{p-1-\delta}{p-1}}\left(\int_{R-\varepsilon}^{R}\left|\widetilde{u}_{r}\right|^{p-1}\left|\widetilde{h}^{\prime}\right| r^{N-1}\right)^{\frac{1}{p-1}},
\end{aligned}
$$

hence

$$
\begin{aligned}
& \int_{R-\varepsilon}^{R}\left|\widetilde{u}_{r}\right|^{p-1}\left|\widetilde{h}^{\prime}\right| r^{N-1} \\
& \quad \geqslant C\left(\frac{R}{R-\varepsilon}\right)^{-\frac{(N-1)(p-1)}{q-1}}(R-\varepsilon)^{-\frac{(N-1)(p-q)}{q-1}} \varepsilon^{\delta+1-p-\frac{(p-1)(\delta+1)}{q-1}}\left(\int_{R-\varepsilon}^{R}|\widetilde{u}|^{q-1} \widetilde{h} r^{N-1}\right)^{\frac{p-1}{q-1}}
\end{aligned}
$$

and

$$
\begin{aligned}
& \int_{0}^{\tau} \int_{R-\varepsilon}^{R} g_{t} \widetilde{u}^{q-1} \widetilde{h} r^{N-1} \\
& \quad+C\left(\frac{R}{R-\varepsilon}\right)^{-\frac{(N-1)(p-1)}{q-1}}(R-\varepsilon)^{-\frac{(N-1)(p-q)}{q-1}} \varepsilon \varepsilon^{\delta+1-p-\frac{(p-1)(\delta+1)}{q-1}} \int_{0}^{\tau} g\left(\int_{R-\varepsilon}^{R}|\widetilde{u}|^{q-1} \tilde{h} r^{N-1}\right)^{\frac{p-1}{q-1}} \\
& \quad+C\left(u_{0} ; \delta, \varepsilon\right) \tau^{\gamma} \leqslant 0 .
\end{aligned}
$$

We have $\int|f|^{1 / r} \leqslant\left(\int|f g|\right)^{1 / r}\left(\int|g|^{-r^{\prime} / r}\right)^{1 / r^{\prime}}$, hence $\int|f g| \geqslant\left(\int|f|^{1 / r}\right)^{r}\left(\int|g|^{-\frac{1}{r-1}}\right)^{-(r-1)}$ for $r>1$. We apply this inverse Hölder inequality to the second summand:

$$
\begin{aligned}
\int_{0}^{\tau}(\tau-t)^{\gamma}\left(\int_{R-\varepsilon}^{R}|\tilde{u}|^{q-1} \tilde{h} r^{N-1}\right)^{\frac{p-1}{q-1}} & \int_{0}^{\tau}\left[(\tau-t)^{\gamma} \int_{R-\varepsilon}^{R}|\widetilde{u}|^{q-1} \tilde{h} r^{N-1}\right]^{\frac{p-1}{q-1}}\left[(\tau-t)^{\gamma}\right]^{-\frac{p-q}{q-1}} \\
& \geqslant\left[\int_{0}^{\tau}(\tau-t)^{\gamma} \int_{R-\varepsilon}^{R}|\widetilde{u}|^{q-1} \widetilde{h} r^{N-1}\right]^{\frac{p-1}{q-1}}\left[\int_{0}^{\tau}(\tau-t)^{\gamma}\right]^{-\frac{p-q}{q-1}} \\
& =(\gamma+1)^{\frac{p-q}{q-1}}\left[\int_{0}^{\tau}(\tau-t)^{\gamma} \int_{R-\varepsilon}^{R}|\widetilde{u}|^{q-1} \tilde{h} r^{N-1}\right]^{\frac{p-1}{q-1}} \tau^{-\frac{(\gamma+1)(p-q)}{q-1}} .
\end{aligned}
$$

Let

$$
F(\tau):=\int_{0}^{\tau}(\tau-t)^{\gamma} \int_{R-\varepsilon}^{R}|\widetilde{u}|^{q-1} \widetilde{h} r^{N-1} \geqslant 0
$$

Then

$$
F^{\prime}(\tau)=\int_{0}^{\tau} \gamma(\tau-t)^{\gamma-1} \int_{R-\varepsilon}^{R}|\widetilde{u}|^{q-1} \widetilde{h} r^{N-1}
$$


and we therefore get the differential inequality

$$
\begin{array}{r}
-F^{\prime}(\tau)+C\left(\frac{R}{R-\varepsilon}\right)^{-\frac{(N-1)(p-1)}{q-1}(R-\varepsilon)^{-\frac{(N-1)(p-q)}{q-1}} \varepsilon^{\delta+1-p-\frac{(p-1)(\delta+1)}{q-1}} \tau^{-\frac{(\gamma+1)(p-q)}{q-1}} F(\tau)^{\frac{p-1}{q-1}}} \\
+C\left(u_{0} ; \delta, \varepsilon\right) \tau^{\gamma} \leqslant 0,
\end{array}
$$

hence

$$
\begin{aligned}
F^{\prime}(\tau) \geqslant & C\left(u_{0} ; \delta, \varepsilon\right) \tau^{\gamma} \\
& +C\left(\frac{R}{R-\varepsilon}\right)^{-\frac{(N-1)(p-1)}{q-1}}(R-\varepsilon)^{-\frac{(N-1)(p-q)}{q-1}} \varepsilon^{\delta+1-p-\frac{(p-1)(\delta+1)}{q-1}} \tau^{-\frac{(\gamma+1)(p-q)}{q-1}} F(\tau)^{\frac{p-1}{q-1}} .
\end{aligned}
$$

Applying Lemma 7 proves the claimed estimate of the waiting time.

COROLlary 10 (Radially symmetric version) Let $A, R, \gamma>0$, and $u: \mathbb{R}^{N} \times[0, \infty) \rightarrow[0, \infty$ ) be a nonnegative radially symmetric weak solution of $(1)$ with initial value $u_{0}(x)=A(R-|x|)_{+}^{\gamma}$ and $\tilde{u}_{r} \leqslant 0$. If $\gamma=p /(p-q)$, then $t_{\Omega}^{\star} \leqslant C A^{-(p-q)}$ with $C=C(p, q)>0$, independent of the spatial dimension. If $\gamma<p /(p-q)$, then $t_{\Omega}^{\star}=0$.

Proof. For any $\varepsilon \in(0, R)$ we have

$$
\begin{aligned}
C\left(u_{0} ; \delta, \varepsilon\right) & =\int_{R-\varepsilon}^{R} \tilde{u}_{0}(r)^{q-1}\left(\varepsilon^{\delta}-(R-r)^{\delta}\right) r^{N-1} \mathrm{~d} r \\
& =A^{q-1} \int_{0}^{\varepsilon} t^{\gamma(q-1)}\left(\varepsilon^{\delta}-t^{\delta}\right)(R-t)^{N-1} \mathrm{~d} t \\
& \geqslant C A^{q-1}(R-\varepsilon)^{N-1} \varepsilon^{\delta+\gamma(q-1)+1} .
\end{aligned}
$$

Apply Theorem 9 to get

$$
t_{\Omega}^{\star} \leqslant C A^{-(p-q)}\left(\frac{R}{R-\varepsilon}\right)^{\frac{(N-1)(p-1)}{q-1}} \varepsilon^{-\frac{(\delta+\gamma(q-1)+1)(p-q)}{q-1}+p-1-\delta+\frac{(p-1)(\delta+1)}{q-1}} .
$$

The exponent of $\varepsilon$ vanishes for $\gamma=p /(p-q)$. Now let $\varepsilon \searrow 0$.

The Main Theorem is a simple consequence:

Proof of Theorem $1 \quad$ We may assume $y_{0}=0$. Let $v_{0}(x):=A\left(r_{0}-|x|\right)_{+}^{\gamma}$. Then we have $v_{0}(x)=$ $A\left(\left|x_{0}\right|-|x|\right)_{+}^{\gamma} \leqslant A\left|x-x_{0}\right|^{\gamma}=u_{0}(x)$ for all $x \in B$. The function $v_{0}$ is radially symmetric with nonpositive radial derivative. By Theorem 6 let $u, v$ be the weak solutions with initial values $u_{0}$ resp. $v_{0}$. Apply Corollary 10 to $v_{0}$.

REMARK 11 This technique also applies to nonnegative radially symmetric weak solutions with nonpositive radial derivative of the equation

$$
\left\{\begin{aligned}
\left(|u|^{q-2} u\right)_{t}-\operatorname{div}\left(|\nabla u|^{p-2} \nabla u\right)-\lambda u^{q-1} & =0 & & \text { in } \mathbb{R}^{N} \times[0, \infty), \\
u(x, 0) & =u_{0}(x) & & \text { for all } x \in \mathbb{R}^{N},
\end{aligned}\right.
$$

with parameters $p \geqslant 2,1<q<p$, and $\lambda>0$. Then for the waiting time one establishes the upper bound $t_{\Omega}^{\star} \leqslant(C / \lambda) \ln \left(1+C \lambda A^{-(p-q)}\right)$ with $C=C(p, q)>0$ if the critical growth exponent for the initial value $u_{0}$ is $\gamma=p /(p-q)$, and $t_{\Omega}^{\star}=0$ if $\gamma<p /(p-q)$. 


\section{REFERENCES}

1. Antontsev, S. N., Díaz, J. I., \& Shmarev, S. Energy Methods for Free Boundary Problems. Progr. Nonlinear Differential Equations Appl. 48, Birkhäuser, Boston (2002). Zbl 0988.35002 MR 1858749

2. CHIPot, M., \& Sideris, T. An upper bound for the waiting time for nonlinear degenerate parabolic equations. Trans. Amer. Math. Soc. 288 (1985), 423-427. Zbl 0535.35049 MR 0773069

3. CHOI, S., \& KIM, I. Waiting time phenomena of the Hele-Shaw and the Stefan problem. Indiana Univ. Math. J. 55 (2006), 525-551. Zbl pre05034334 MR 2225444

4. Giacomelli, L., \& GRÜN, G. Lower bounds on waiting time for degenerate parabolic equations and systems. Interfaces Free Bound. 8 (2006), 111-129. Zbl 1100.35058 MR 2231254

5. Heinig, H. P., \& Kufner, A. Hardy's inequality for higher order derivatives. Proc. Steklov Inst. Math. 3 (1992), 113-121. Zbl 0789.26010 MR 1097892

6. ISHIGE, K. On the existence of solutions of the Cauchy problem for a doubly nonlinear parabolic equation. SIAM J. Math. Anal. 27 (1996), 1235-1260. Zbl $0858.35056 \mid$ MR 1402438

7. Ladyženskaja, O. A., Solonnikov, V. A., \& Ural'Ceva, N. N. Linear and Quasi-linear Equations of Parabolic Type. Transl. Math. Monogr. 23, Amer. Math. Soc., Providence, RI (1988). Zbl 0174.15403 MR 0241822

8. Protter, M. H., \& Weinberger, H. F. Maximum Principles in Differential Equations. Prentice-Hall, Englewood Cliffs, NJ (1967). Z Zbl 0153.13602 MR 0219861

9. Shishrov, A. E., \& Shchelkov, A. G. Dynamics of the supports of energy solutions of mixed problems for quasi-linear parabolic equations of arbitrary order. Izv. Math. 62 (1998), 601-626. Zbl 0918.35075 MR 1642176

10. Sinnamon, G., \& Stepanov, V. D. The weighted Hardy inequality: new proofs and the case $p=1$. J. London Math. Soc. 54 (1996), 89-101. Zbl 0856.26012 MR 1395069

11. Tsutsumi, M. On solutions of some doubly nonlinear degenerate parabolic equations with absorption. J. Math. Anal. Appl. 132 (1988), 187-212. Zbl $0681.35047 \mid$ MR 0942364 